\title{
MicroRNA-149 Suppresses Colorectal Cancer Cell Migration and Invasion by Directly Targeting Forkhead Box Transcription Factor FOXM1
}

\author{
Kai Xu ${ }^{a} \quad$ Xiaobei Liu ${ }^{b} \quad$ Xiaobei Mao ${ }^{b}$ Lijun Xue ${ }^{b} \quad$ Rui Wang $^{b} \quad$ Longbang Chen $^{b}$ \\ Xiaoyuan Chu ${ }^{a, b}$ \\ aDepartment of Medical Oncology, Jinling Hospital, Nanjing Clinical School of Southern Medical \\ University, 'Department of Medical Oncology, Jinling Hospital, School of Medicine, Nanjing University, \\ Nanjing, Jiangsu, PR China
}

\section{Key Words}

miR-149 • FOXM1 • Migration • Invasion • Colorectal cancer

\begin{abstract}
Background/Aims: The aim of this study is to investigate the clinicopathological and prognostic values of miR-149 expression and its roles in colorectal cancer (CRC) progression. Methods: qRT-PCR was performed to detect miR-149 expression in CRC cell lines or tissues. Also, the clinical significance of miR-149 expression was investigated. The study further explored whether miR-149 inhibits migration and invasion of CRC cells by targeting the mammalian Forkhead Box M1 (FOXM1). Results: miR-149 was significantly downregulated in CRC tissues, and low miR-149 expression was observed to be significantly correlated with lymph node or distant metastasis and advanced TNM stage of CRC patients. Patients with low miR-149 expression showed poorer prognosis than those with high miR-149 expression, and multivariate analyses indicated that status of miR-149 expression might be an independent prognostic factor. Gain- and loss - of - function assays indicated that miR-149 significantly inhibited growth, migration and invasion of CRC cells by targeting FOXM1. Furthermore, FOXM1 was significantly upregulated in CRC tissues and inversely correlated with miR-149 expression. Conclusions: miR-149 was an independent prognostic factor and could inhibit migration and invasion of CRC cells, at least partially by targeting FOXM1.
\end{abstract}




\section{Cellular Physiology and Biochemistry}

Cell Physiol Biochem 2015;35:499-515

\begin{tabular}{l|l}
\hline DOI: $10.1159 / 000369715$ & (C) 2015 S. Karger AG, Basel
\end{tabular}

Xu et al.: MiR-149 Inhibits Tumor Cell Migration and Invasion by Targeting FOXM1 in CRC

\section{Introduction}

Colorectal cancer (CRC) is the third leading cause of cancer-related deaths worldwide, and its overall incidence is $\sim 5 \%$ and the 5 years survival rate ranges from 40 to $60 \%$ [1]. Many progresses made in the treatment of CRC in the past decades, but the overall survival of patients with CRC is still low. Poor prognosis and survival rate are mainly due to metastasis, thus more than one-third of patients with CRC will ultimately develop metastatic diseases [2]. Due to the fact that the molecular mechanisms of CRC cell migration and invasion are highly complex, further identification of new candidate molecules that take part in these processes is crucial for developing targeted therapy for CRC.

MicroRNAs (miRNAs), a class of 17-25 nucleotides small non-coding RNAs, associate with 3'-untranslated regions (3'-UTR) of specific target messenger RNAs (mRNAs) to suppress translation and occasionally also induce their degradation [3]. Dsyregulated miRNAs play critical roles in many human physiological and pathological processes, such as cellular growth, differentiation, apoptosis, development, malignant transformation and metastasis [4-6]. Recently, miR-149 is reported to be downregulated in many human cancers and functions as a tumor suppressor in non-small cell lung cancer, glioma and gastric cancer [7-9]. The correlations of miR-149 with CRC are also reported. Wang, et al showed that SP1 mediates the link between methylation of the tumour suppressor miR-149 and outcome in CRC [10]. Vinci and his colleagues investigated the distribution of sequence variants of miR-146a, miR-196a, miR-499 and miR-149 in colorectal cancer (CRC) and their effects on miRNA expression [11]. Also, Du and his colleagues showed that the miR-149 rs2292832 and miR-196a2 rs11614913 polymorphisms may contribute to susceptibility to CRC [12]. These results indicated that dysregulation of miR-149 might play an important role in CRC progression and development. Although it has been reported that miR-149 inhibits nonsmall cell lung cancer cells EMT by targeting FOXM1 [13], whether miR-149 targets FOXM1 to suppress CRC migration and invasion is not unclear and will be investigated in this report.

In the present study, we first investigated the expression of miR-149 in CRC cell lines and tissue samples, and analyzed its clinicopathological and prognostic values. By gain and loss - of - function studies, we identified miR-149 as a negative regulator of migration and invasion of CRC cells through post-transcriptionally downregulating FOXM1, which is a master regulator of tumor metastasis. Thus, target miR-149/FOXM1 signaling will be a potential strategy for the treatment of metastatic CRC.

\section{Materials and Methods}

\section{Cell culture}

Human colorectal cancer cell lines (HCT116, LoVo and SW480) and a normal colonic cell line (NCM460) were cultured at at $37^{\circ} \mathrm{C}$ in RPMI-1640 medium supplemented with $10 \%$ calf serum, $100 \mathrm{U} / \mathrm{mL}$ penicillin, and $100 \mu \mathrm{g} / \mathrm{mL}$ streptomycin, in an atmosphere of $5 \% \mathrm{CO}_{2}$ at saturation humidity. Cells in logarithmic growth were used in all experiments.

\section{Patients and tissue samples}

A total of 78 cases of CRC tissues, 20 paired of CRC and the adjacent normal tissues, and 20 cases of normal colon tissues were collected directly from surgery after removal of the necessary amount of tissue at the Department of Pathology or Surgery, Jinling or Jiangsu Provin Hospital between 2006 and 2008. Histopathological analyses confirmed the malignant and adjacent normal colon tissues. Tumor staging was defined according to the criteria for histological classification proposed by the International Union Against Cancer (UICC). Patients were informed of the investigational nature of the study and each provided written informed consent prior to recruitment. None of the patients had received chemotherapy or radiation therapy. For RT-PCR and Western blot examination, all tissues were immediately frozen in liquid nitrogen and stored at $-70^{\circ} \mathrm{C}$ until use. 


\section{Cellular Physiology \\ and Biochemistry}

Cell Physiol Biochem 2015;35:499-515

\begin{tabular}{l|l}
\hline DOI: $10.1159 / 000369715$ & (c) 2015 S. Karger AG, Basel
\end{tabular}

www.karger.com/cpb

Xu et al.: MiR-149 Inhibits Tumor Cell Migration and Invasion by Targeting FOXM1 in CRC

Taqman quantitative reverse transcription ( $q R T$ )-PCR of miRNA

Total RNA isolation from tissues was performed using mirVana miRNA Isolation Kit (Ambion, Austin, TX, USA). RNA concentrations were measured using the SPECTRAmax microplate spectrophotometer (Molecular Devices Corp). The cDNA was synthesized from 5 ng of total RNA by using the Taqman miRNA reverse transcription kit (Applied Biosystems, Foster City, CA), and the expression levels of miR-149 were quantified by using miRNA-specific TaqMan MiRNA Assay Kit (Applied Biosystems). qRT-PCR was performed by using the Applied Biosystems 7500 Sequence Detection system. The expression of miRNA was defined based on the threshold cycle (Ct), and relative expression levels were calculated as $2^{-[(\mathrm{Ct} \text { of } 149) \text {-(Ct of U6)] }}$ after normalization with reference to expression of U6 small nuclear RNA.

Quantitative real-time PCR of $m R N A s$

To measure the relative expression of mRNAs, two micrograms of total RNAs of each sample were used for RT reaction in $20 \mu \mathrm{L}$ of reaction volume, using a reverse transcription system (Invitrogen, CA, USA). Quantitative real-time PCR was carried out using realtime PCR with the SYBR Green reporter following manufacturer's protocol. Sequences of PCR primers (MMP-2, MMP-9, VEGF-A and UPA) were described previously $[13,14]$. Real-time PCR reactions were performed in $25 \mu \mathrm{L}$ of reaction mixture using a Smart Cycler II thermocycler. Data were analyzed using Ct value and were normalized by the expression of control mRNA GAPDH in each sample.

Western blotting assay

The cells were lysed using modified radioimmuno precipitation assay buffer (50mM Tris- $\mathrm{HCl}(\mathrm{pH}$ 7.4), $1 \%$ NP-40, $0.25 \%$ sodium deoxycholate, $150 \mathrm{mM} \mathrm{NaCl}, 1 \mathrm{mM}$ ethylenediaminetetraacetic acid, protease inhibitor cocktail complete. Amounts of total protein extracts were determined using BCA assay and samples were stored at $-134^{\circ} \mathrm{C}$ until use. Proteins were separated by sodium dodecyl sulfate-polyacrylamide gel electrophoresis (SDS-PAGE). Probing and detection of specific proteins was performed with enhanced chemiluminescence (ECL) after antibody binding. The following antibodies are used: anti-FOXM1, MMP-2, MMP-9, VEGF-A, UPAR and GAPDH (Santa Cruz Biotechnology, Santa Cruz, CA, USA). The membranes were then incubated with a horseradish peroxidase-conjugated secondary antibody (Sigma, USA). The proteins were detected by an enhanced chemiluminescence detection system, and light emission was captured on Kodak X-ray films.

\section{Transfection of miRNA mimics, inhibitors or plasmid vectors}

MiR-149 mimics or inhibitor (anti-miR-149) and their negative control oligonucleotides (miR-NC mimics or anti-miR-NC) were obtained from Ambion Inc (Austin, TX, USA). The small hairpin RNA (shRNA) plasmid vector targeting FOXM1 (pSil/shFOXM1) and control vector (pSil/shcontrol) were successfully constructed previously [15]. The open reading frame of FOXM1 that was generated by PCR was then inserted into the pEGFP-C1 expression vector which was named pEGFP/FOXM1. The recombinant vector was confirmed by the digestion analysis of restriction endonuclease and DNA sequencing. The transfection were performed using Lipofectamin $\mathrm{e}^{\mathrm{TM}} 2000$ (Invitrogen, USA) according to the instructions provided by the manufacturer. The cells were transfected with those recombinant DNA vectors containing a G418 selection marker and were selected G418 (Sigma) at $400 \mathrm{mg} / \mathrm{ml}$ for 4 weeks Then, single clones were obtained and maintained in G418 at $100 \mathrm{mg} / \mathrm{ml}$.

\section{3-(4,5-dimethylthazol-2-yl)-2,5-diphenyltetrazolium bromide (MTT) assay}

The cells in $100 \mu \mathrm{L}$ of the medium were plated in 96-well plates. Every 24 hours up to 7 days, the medium was replaced with $100 \mu \mathrm{L}$ of $0.05 \%$ MTT/PBS (-). Then, the cells were incubated at $37^{\circ} \mathrm{C}$ for $4 \mathrm{~h}$, the reaction was stopped by the addition of $200 \mathrm{ml} /$ well of DMSO, lysing for $10 \mathrm{~min}$. The protracted cell growth curve and the results of the inhibitory rates of cell growth were applied to absorbance (A) at $540 \mathrm{~nm}$ using Versamax microplate reader (Molecular Devices, Sunnyvale, CA).

\section{Wound healing assay}

The cell were implanted in the culture dishes. When the cells grown to $80 \%$ confluence, a sterilized tip was used to draw a line with the same width on the bottom of $35 \mathrm{~mm}$ dishes. Photos were taken at 0 or $48 \mathrm{~h}$ after the wounding. 


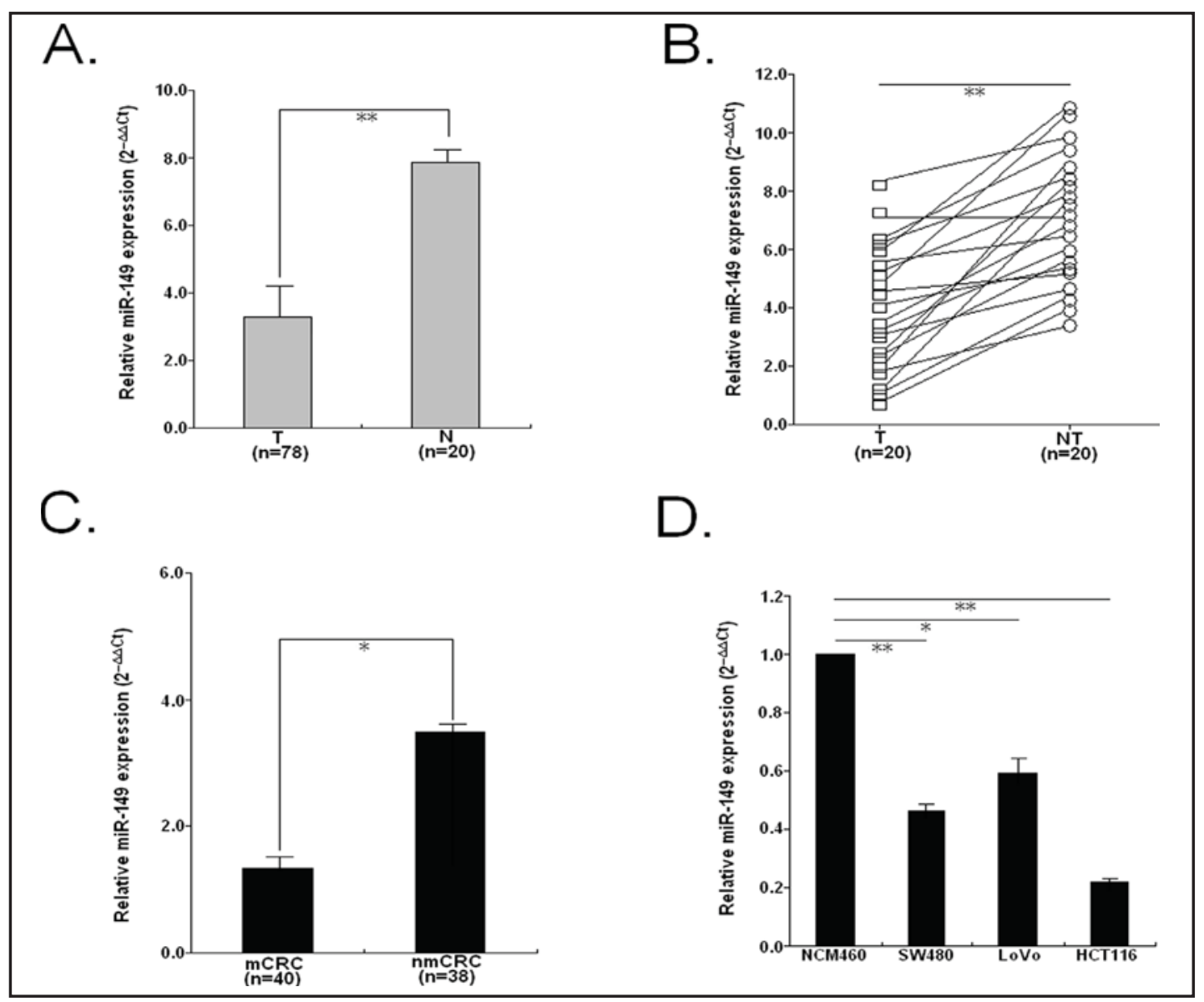

Fig. 1. The expression of miR-149 was significantly downregulated in CRC cell lines and tissue samples by qRT-PCR. miRNA abundance was normalized to U6 RNA. (A) The relative expression level of mature miR149 in CRC tissues ( $n=78)$ and normal colon tissues $(n=20)$. (B) The relative expression level of mature miR149 in 20 paired of CRC and the adjacent normal colon tissues. (C) The relative expression level of mature miR-149 in CRC tissues with lymph node or distant metastasis (mCRC, n=40) or without metastasis ( $\mathrm{nm}$ CRC, $n=38$ ). (D) The relative expression of miR-149 in CRC cell lines (SW480, LoVo and HCT116) and normal colonic cell line (NCM460). Results represent the average of three independent experiments (mean \pm SD). ${ }^{*} P<0.05,{ }^{* *} P<0.01$.

\section{Transwell cell invasion assay}

In vitro invasion assay was determined using matrigel invasion chambers (BD Bioscience, San Diego, CA, USA). The cells were seeded into inserts at $2.0 \times 10^{4}$ per insert in serum-free medium and then transferred to wells filled with the culture medium containing 10\% FBS (Sijiqing Biotech, PR China) as a chemoattractant. After $24 \mathrm{~h}$ of incubation, non-invading cells on the top of the membrane were removed by scraping. Invaded cells on the bottom of the membrane were fixed, followed by staining with $0.05 \%$ crystal violet. The number of invaded cells on the membrane was then counted under a microscope.

\section{Statistical assay}

For continuous variables, data are expressed as mean \pm standard deviation (SD). The Chi-squared test was used to investigate the significance of miR-149 expression as correlated with clinicopathologic factors in CRC. The overall survival (OS) curves were plotted using the Kaplan-Meier method and were evaluated for the statistical significance using a log-rank test. The significance of different variables with respect to survival was analyzed using the univariate and multivariate Cox proportional hazards model. Differences were considered statistically significant when $P<0.05$. Statistical analysis was performed using SPSS 17.0 software program (SPSS Inc., Chicago, Illinois, USA). 
Table 1. Correlation of miR-149 expression with clinicopathological factors. of CRC patients. ${ }^{*} P<0.05$ was considered statistically significant

\begin{tabular}{|c|c|c|c|}
\hline Factors & $\begin{array}{l}\text { Low-miR-149 } \\
\text { expression }(n=45) \\
\text { Number }(\%)\end{array}$ & $\begin{array}{l}\text { High-miR-149 } \\
\text { expression }(\mathrm{n}=33) \\
\text { Number }(\%)\end{array}$ & $P$-value \\
\hline Age (years) & & & 0.581 \\
\hline$\geq 65$ & $15(33.3)$ & $13(39.4)$ & \\
\hline$<65$ & $30(66.7)$ & $20(60.6)$ & \\
\hline Gender & & & 0.583 \\
\hline Male & $26(57.8)$ & $17(51.5)$ & \\
\hline Female & $19(42.2)$ & $16(48.5)$ & \\
\hline Tumor site & & & 0.276 \\
\hline Colon & $32(71.1)$ & $27(81.8)$ & \\
\hline Rectum & $13(28.9)$ & $6(18.2)$ & \\
\hline Tumor size & & & 0.859 \\
\hline$<5.0 \mathrm{~cm}$ & $20(44.4)$ & $14(42.4)$ & \\
\hline $25.0 \mathrm{~cm}$ & $25(55.6)$ & $19(57.6)$ & \\
\hline Tumor differentiation & & & 0.524 \\
\hline Well/Moderate & $12(26.7)$ & $11(33.3)$ & \\
\hline Poor & $33(73.3)$ & $22(66.7)$ & \\
\hline Lymph node metastasis & & & $0.024^{\circ}$ \\
\hline No & $17(37.8)$ & $21(63.6)$ & \\
\hline $\mathrm{N} 1+2$ & $28(62.2)$ & $12(36.4)$ & \\
\hline Distant metastasis & & & $0.045^{\circ}$ \\
\hline No & $29(64.4)$ & $28(84.8)$ & \\
\hline Yes & $16(35.6)$ & $5(15.2)$ & \\
\hline TNM stage & & & $0.033^{\circ}$ \\
\hline I+II & $19(42.2)$ & $22(66.7)$ & \\
\hline $\mathrm{III}+\mathrm{IV}$ & $26(57.8)$ & $11(33.3)$ & \\
\hline
\end{tabular}

\section{Results}

MiR-149 is significantly downregulated in metastatic CRC tissues and a poor prognostic factor for patients

First, qRT-PCR assay was performed to detect the expression of miR-149 in 78 CRC tissue samples and 20 normal colon tissues, which is normalized to U6 RNA. As shown in Fig. $1 \mathrm{~A}$, the relative expression level of miR-149 in CRC tissues was significantly lower than that in normal colon tissues $(P<0.01)$. In comparison with 20 paired normal colon tissues, the relative expression level of miR-149 was also significantly lower in the tumor tissues $(P<0.01$; Fig. 1B). Interestingly, we showed that the relative expression level of miR-149 in CRC tissues with lymph node metastasis or distant metastasis ( $m C R C, n=40$ ) was significantly lower than that in tumor tissues without metastasis (nmCRC, $\mathrm{n}=38)(P<0.01$; Fig. $1 \mathrm{C})$. Then, we detected the expression of miR-149 in three CRC cell lines (SW480, LoVo and HCT116) and the normal colonic cell line (NCM460), and showed that miR-149 was significantly downregulated in CRC cell lines in comparison with the normal colonic cell line (NCM460). Also, among the three CRC cell lines, HCT116 (high-metastatic potential) exhibited the lowest miR-149 level (Fig. 1D).

To further investigate the clinical significance of miR-149 in CRC, we divided the 78 patients into two groups according to the median value (3.36) of the miR-149 expression level in CRC tissues: high-miR-149 group $(n=33)$ and low-miR-149 group $(n=45)$. By statistical analysis, the expression level of miR-149 was observed to be significantly correlated with lymph node metastasis, distant metastasis and TNM stage $(P=0.024,0.045$ and 0.033 , respectively), but not with other clinicopathological factors, such as age, gender, tumor site, tumor size and tumor differentiation $(P=0.581,0.583,0.276,0.859$ and 0.524 , respectively) (Table. 1). Then, Kaplan-Meier survival analysis was performed to analyze the correlation 
Table 2. Multivariate analyses for prognostic factors in CRC patients. HR: hazard ratio; 95\% CI: 95\% confidence interval; ${ }^{*} P<0.05$

\begin{tabular}{llll}
\hline Clinicopathological factor & $P$-value & HR & $95 \% \mathrm{CI}$ \\
\hline Age $(>65$ v $\leq 60)$ & 0.145 & 1.088 & $0.498-1.356$ \\
Gender (Male $v s$ Female) & 0.209 & 0.897 & $0.512-1.268$ \\
Tumor site (Colon $v s$ Rectum) & 0.177 & 1.344 & $0.897-1.935$ \\
Tumor size ( $\geq 5.0 \mathrm{~cm}$ vs $<5.0 \mathrm{~cm}$ ) & 0.523 & 0.936 & $0.654-1.434$ \\
Tumor differentiation (Poor $v s$ Well/Modertate) & 0.378 & 1.889 & $0.983-2.304$ \\
Lymph node metastasis $\left(\mathrm{N}_{1+2}\right.$ vs $\mathrm{N}_{0}$ ) & 0.035 & 2.487 & $1.729-3.006$ \\
Distant metastasis (Yes $v s$ No) & $0.014^{*}$ & 2.046 & $1.723-2.808$ \\
TNM stage (III+IV $v s$ I+II) & $0.008^{*}$ & 3.457 & $1.203-4.016$ \\
MiR-149 expression (Low vs High) & $0.011^{*}$ & 2.658 & $1.308-3.087$ \\
\hline
\end{tabular}

Fig. 2. Kaplan-Meier overall survival curves of LAD patients. The overall survival (OS) probability in CRC patients with low-miR-149 expression $(n=45)$ was significantly lower than that in patients with highmiR-149 expression $(n=33)$. Log rank test, $P=0.0018$.

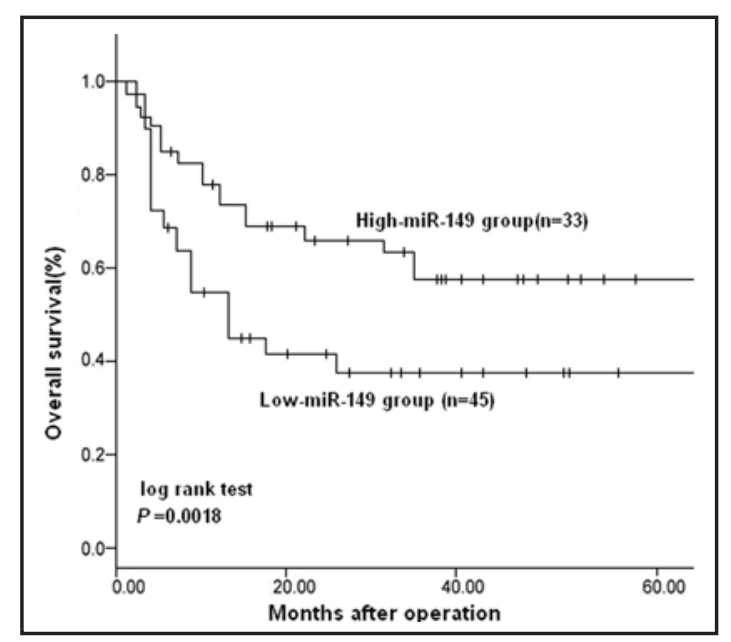

of miR-149 expression with prognosis of CRC patients (Fig. 2). Patients with low-miR-149 expression exhibited significantly lower over survival (OS) rate than those with high-miR-149 expression, and the five-year survival rate for patients with low-miR-149 expression was $25.5 \%$, compared with $42.3 \%$ for those patients with high-miR-149 expression $(P=0.0018)$. Furthermore, multivariate analysis based on the Cox proportional hazards regression model showed that low miR-149 expression might be an independent prognostic factor for CRC patients ( $P=0.011$; HR=2.658; 95\% CI: 1.308-3.087), in addition to lymph node or distant metastasis, and TNM stage (Table. 2). These data suggested that downregulation of miR-149 might play an important role in CRC progression and metastasis.

\section{MiR-149 inhibits migration and invasion of CRC cells}

To further determine the effects of miR-149 on malignant phenotypes of CRC cells, LoVo cells with high miR-149 expression were transfected with the miR-149 inhibitor to knockdown endogenous miR-149, whereas HCT116 cells with low miR-149 expression were transfected with the miR-149 mimics to overexpress miR-149, which were confirmed by qRT-PCR assay (Fig. 3A). First, we performed MTT assay to analyze the effect of miR-149 on growth of CRC cells, and showed that upregulation of miR-149 could moderately increase the growth of LoVo cells and downregulation of miR-149 could significantly reduce the growth of HCT-116 cells (Fig. 3B). Also, since miR-149 was observed to be significantly downregulated in CRC tissues with metastasis, we next determine the effects of miR-149 expression in migration and invasion of CRC cells. The wound healing assay indicated that upregulation of miR-149 could induce a slower closing of scratch wounds in HCT116 cells and downregulation of miR-149 could induce a faster closing of scratch wounds in LoVo cells (Fig. 3C). Similarly, in Matrigel invasion assays (Fig. 3D), upregulation of miR-149 could significantly reduce 


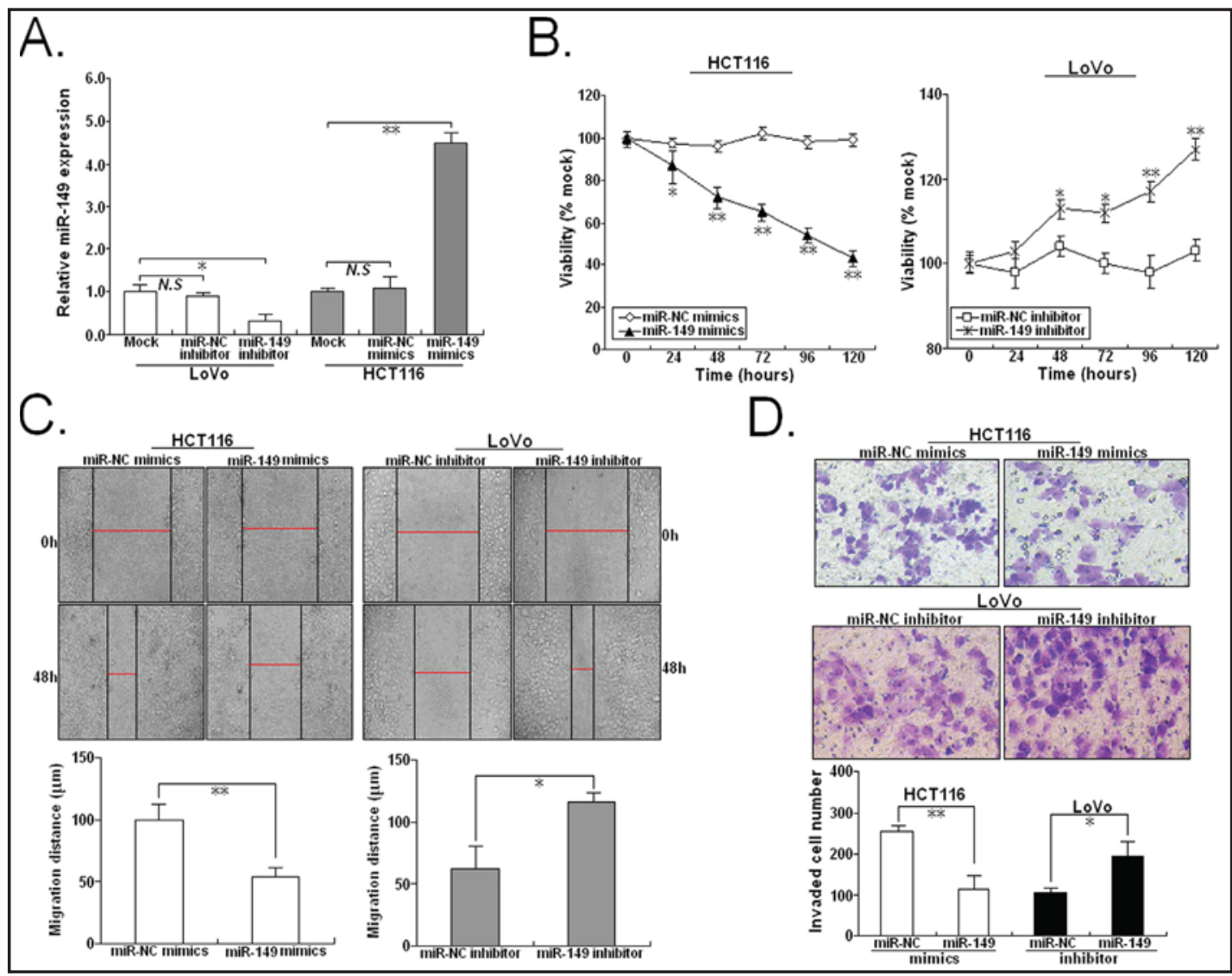

Fig. 3. miR-149 significantly inhibits growth, migration and invasion of CRC cells. miRNA abundance was normalized to U6 RNA. (A) qRT-PCR detection of miR-149 expression in mock, miR-NC mimics or miR-149 mimics-transfected HCT116 or mock, anti-miR-NC or anti-miR-149 -transfected LoVo cells. U6 RNA was used as an internal control. (B) MTT analysis of growth in miR-NC or miR-149 mimics-transfected HCT116 or miR-NC or miR-149 inhibitor-transfected LoVo cells. The cell viability was then analyzed daily over $120 \mathrm{~h}$, and mock cells were used as a control in each point. (C) Effect of miR-149 expression on migration of CRC cells using wound scratch healing assay. (D) Effect of miR-149 expression on invasion of CRC cells using Transwell invasion assay. Results represent the average of three independent experiments (mean \pm SD). ${ }^{*} P<0.05,{ }^{* *} P<0.01$. N.S: not significance versus control.

invasion of HCT116 cells (118 versus 258, $P<0.01$ ). Likewise, downregulation of miR-149 could moderately increase invasion of LoVo cells (188 versus $104, P<0.05$ ). Interestingly, it was also observed that transfection of anti-miR-149 could lead to the moderately increased ability of migration and invasion in normal colonic cell line (NCM460) (Fig. 4A-C). These data suggested that miR-149 inhibits migration and invasion of CRC cells.

\section{FOXM1 was identified as a direct target of miR-149 in CRC}

The mammalian Forkhead Box (Fox) transcription factor FOXM1 has been reported to be implicated in tumorigenesis [14]. Previously, we have shown that FOXM1 overexpression is a molecular marker predicting increased invasive/metastatic potential of CRC and a poorer prognosis [15]. However, the molecular mechanism involved in FOXM1 overexpression in CRC is not clear. MiRNAs function mainly through the inhibition of target genes, the targets of miR-149 that function in CRC cells were further investigated. By three databases (Microinspector, Pictarget, and TargetScan), FOXM1 was selected as a putative target of miR-149, which was recently reported in non-small cell lung cancer [13]. Then, the targets of miR-149 were predicted through at least three publicly available algorithms (TargetScan, PicTar and miRanda), and in silico analysis revealed 3'-UTR of human FOXM1 


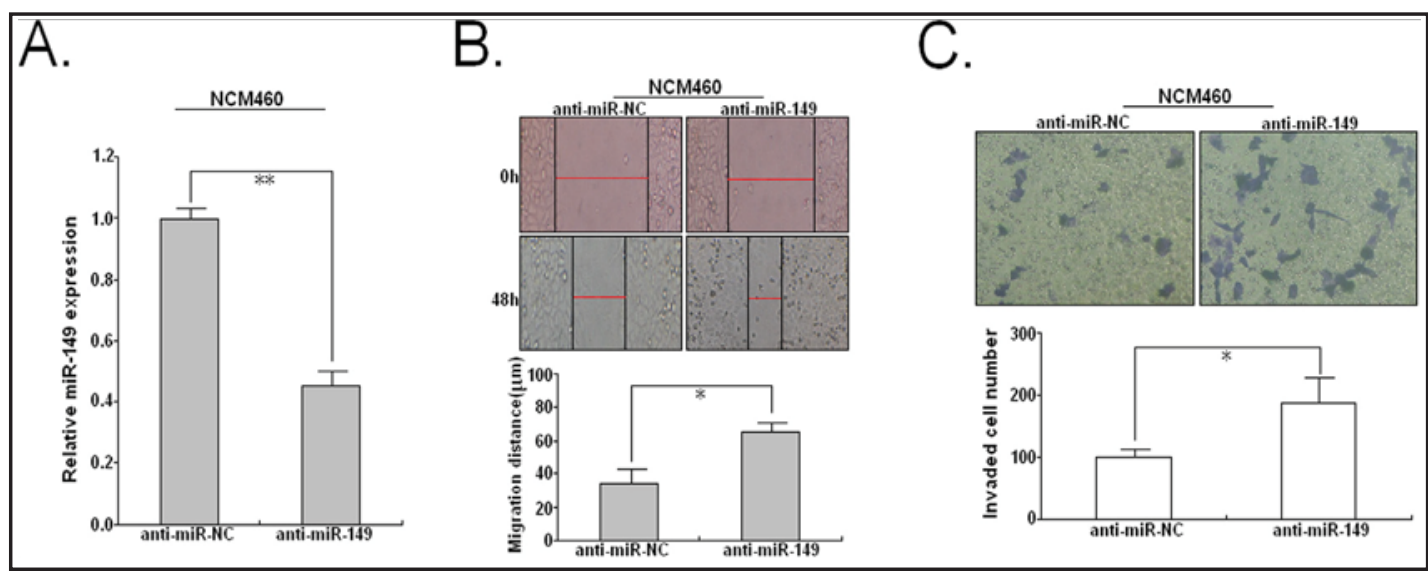

Fig. 4. Effect of miR-149 expression on migration and invasion of normal colonic cell line (NCM460). (A) $48 \mathrm{~h}$ after NCM460 cell line was transiently transfected with anti-miR-149 or anti-miR-NC, qRT-PCR was performed to detect the expression of miR-149 and miRNA abundance was normalized to U6 RNA. (B) Wound scratch healing analysis of migration in anti-miR-149 or anti-miR-NC-transfected NCM460 cells. (D) Transwell invasion analysis of anti-miR-149 or anti-miR-NC-transfected NCM460 cells. Results represent the average of three independent experiments (mean \pm SD). ${ }^{*} P<0.05,{ }^{* *} P<0.01$.

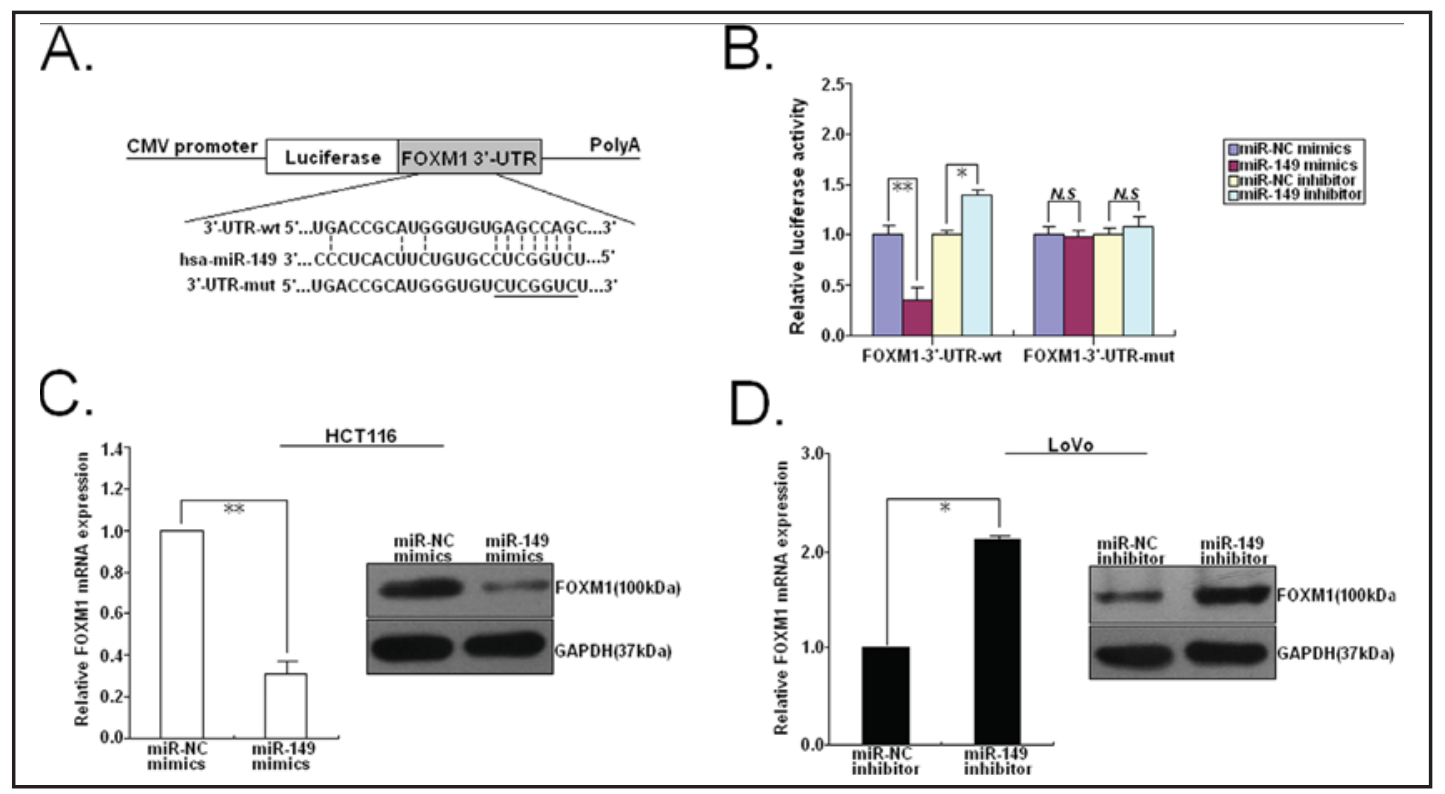

Fig. 5. Identification of FOXM1 as a direct target of miR-149 in CRC cells. (A) miR-149 and its putative binding sequence in the 3'-UTR of FOXM1. The mutant miR-149-binding site waS generated in the complementary site for the seed region of miR-149. (B) Relative luciferase activity was analyzed. pLUC/FOXM13'-UTR-wt or pLUC/FOXM1-3'-UTR-mut vector and miR-149 mimics (or miR-NC mimics) or miR-149 inhibitor (or miR-NC inhibitor) were co-transfected into HCT116 cells, Cells lysates were prepared after 48 h for measuring luciferase activity, which was normalized to normalized to Renilla luciferase activity. (C) qRT-PCR and Western blotting detection of FOXM1 mRNA and protein expression in miR-NC or miR-149 mimics-transfected HCT116 cells. (D) qRT-PCR and Western blotting detection of FOXM1 mRNA and protein expression in miR-NC or miR-149 inhibitor-transfected LoVo cells. GAPDH was used as an internal control. Results represent the average of three independent experiments (mean \pm SD). ${ }^{*} P<0.05$, ${ }^{*} P<0.01$. N.S: not significance versus control.

(3583 3605nt) contains a potential miR-149 binding site. The 3'-UTR sequence of FOXM1 (3'UTR-wt) or the mutant sequence (3'-UTR-mut) was cloned into a luciferase reporter vector pLUC (Fig. 5A). A luciferase reporter assay was performed to determine whether miR- 
A.
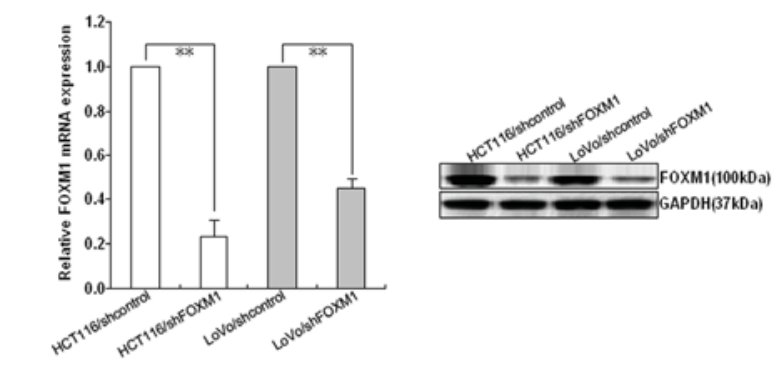

C.
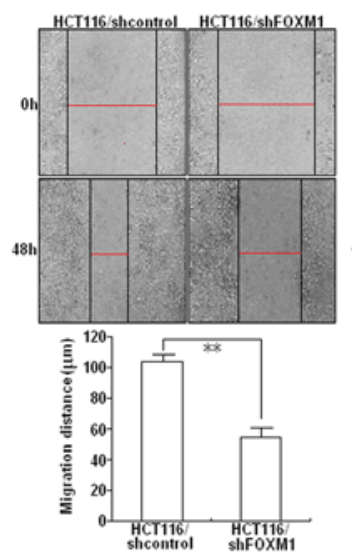

B.

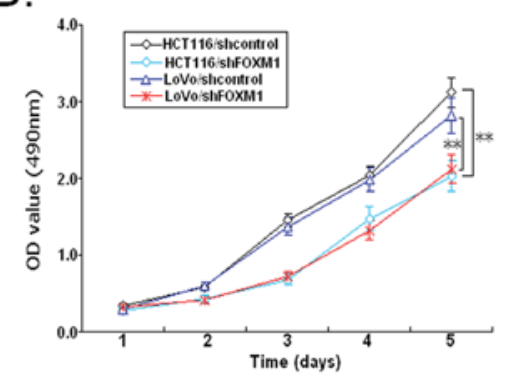

D.

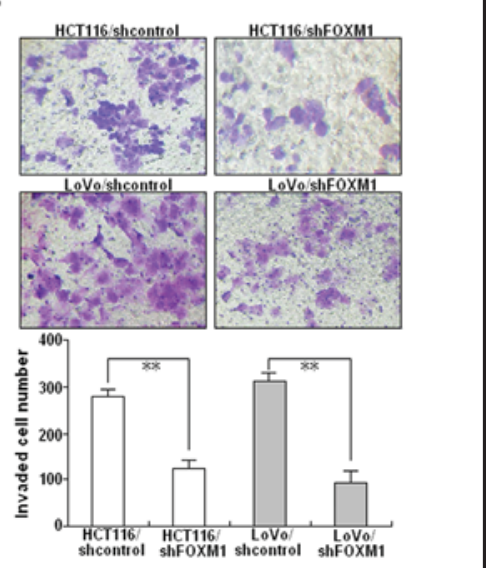

Fig. 6. Silencing of FOXM1 mimics the effects of miR-149 upregulation on phenotypes of CRC cells. (A) qRT-PCR and Western blotting detection of FOXM1 mRNA and protein expression in HCT116 and LoVo cells stably transfected with pSil/shFOXM1 or pSil/shcontrol vector. GAPDH was used as an internal control. (B) MTT analysis of growth in HCT116/shFOXM1 (or HCT116/shcontrol) and LoVo/shFOXM1 (or LoVo/shcontrol) cells. (C) Wound scratch healing analysis of migration in HCT116/shFOXM1 (or HCT116/shcontrol) and LoVo/shFOXM1 (or LoVo/shcontrol) cells. (D) Transwell invasion analysis of HCT116/shFOXM1 (or HCT116/shcontrol) and LoVo/shFOXM1 (or LoVo/shcontrol) cells. Results represent the average of three independent experiments (mean \pm SD). ${ }^{*} P<0.05,{ }^{* *} P<0.01$. N.S: not significance versus control.

149 can directly regulate the expression of FOXM1 in HCT116 cells (Fig. 5B). The results indicated a significant decrease in the luciferase activity of pLUC/FOXM1-3'-UTR-wt which was induced by miR-149 mimics when compared with miR-NC mimics $(P<0.01)$. Compared with anti-miR-NC, the transfection with anti-miR-149 in HCT116 cells led to a significant increase in luciferase activity $(P<0.05)$. However, the activity of pLUC/FOXM1-3'-UTR-mut vector was unaffected with a simultaneous co-transfection with miR-149 mimics or inhibitor $(P>0.05)$. Next, we analyzed the changes of FOXM1 expression in CRC cells after the ectopic overexpression or silencing of miR-149, and showed that upregulation of miR-149 could significantly reduce the expression of FOXM1 mRNA and protein expression in HCT116 cells $(P<0.01$; Fig. 5C) and silencing of miR-149 could lead to the upregulated expression of FOXM1 mRNA and protein in LoVo cells $(P<0.01$; Fig. 5D). These data suggest that FOXM1 is a direct target of miR-149 in CRC cells.

Silencing of FOXM1 significantly inhibits migration and invasion of CRC cells

To further investigate the effects of FOXM1 expression on migration and invasion of CRC cells, pSil/shFOXM1 was stably tansfected into CRC cells (HCT116 and LoVo) and used to knockdown the expression of FOXM1 in CRC cells (HCT116/shFOXM1 and LoVo/ shFOXM1), which were confirmed by qRT-PCR and Western blotting assays $(P<0.05$; Fig. 6A). Next, results from MTT assay indicated that silencing of FOXM1 could lead to the decreased capacity of growth in HCT116 and LoVo cells (Fig. 6B). Also, wound healing and Martrigel 


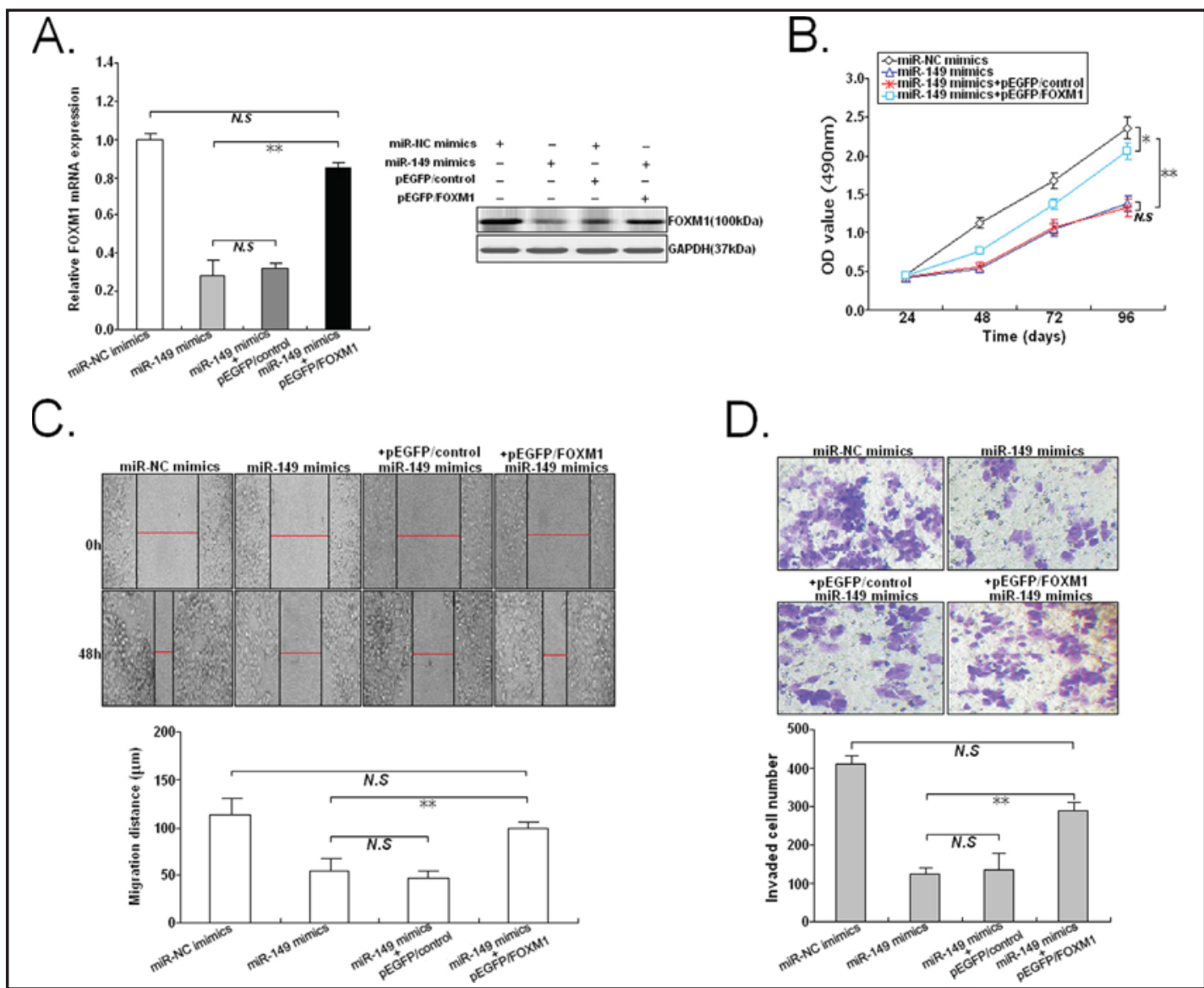

Fig. 7. Overexpression of FOXM1 partially reverses the effects of miR-149 upregulation on phenotypes of CRC cells. (A) qRT-PCR and Western blotting detection of FOXM1 mRNA and protein expression in miR-NC or miR-149 mimics-transfected HCT116 cells or HCT116 cells co-transfected with miR-149 mimics and pEGFP/FOXM1 or pEGFP/control. GAPDH was used as an internal control. (B) MTT analysis of growth in iRNC or miR-149 mimics-transfected HCT116 cells or HCT116 cells co-transfected with miR-149 mimics and pEGFP/FOXM1 or pEGFP/control. (C) Wound scratch healing analysis of migration in miR-NC or miR-149 mimics-transfected HCT116 cells or HCT116 cells co-transfected with miR-149 mimics and pEGFP/FOXM1 or pEGFP/control. (D) Transwell invasion analysis of invasion in miR-NC or miR-149 mimics-transfected HCT116 cells or HCT116 cells co-transfected with miR-149 mimics and pEGFP/FOXM1 or pEGFP/control. Results represent the average of three independent experiments (mean \pm SD). ${ }^{*} P<0.05$, ${ }^{*} P<0.01$. N.S: not significance versus control.

invasion assays indicated that silencing of FOXM1 could significantly reduce the capacity of migration and invasion in CRC cells (Fig. 6C and D). These results clearly indicate that silencing of FOXM1 mimics the effects of miR-149 overexpression on malignant phenotypes of CRC cells.

Overexpression of FOXM1 partially reverses the effects of miR-149 upregulation on malignant phenotypes of CRC cells

To further determine the roles of FOXM1 in phenotypical changes of CRC cells induced by miR-149, HCT116 cells were co-transfected with miR-149 mimics (or miR-NC mimics) and pEGFP/FOXM1 vector. $48 \mathrm{~h}$ after co-tranfection, qRT-PCR and Western blotting assays were performed to detect the expression of FOXM1 mRNA and protein, and results showed that the co-transfection could rescue the decreased expression of FOXM1 mRNA and protein in HCT116 cells induced by miR-149 mimics (Fig. 7A). It was observed that overexpression 


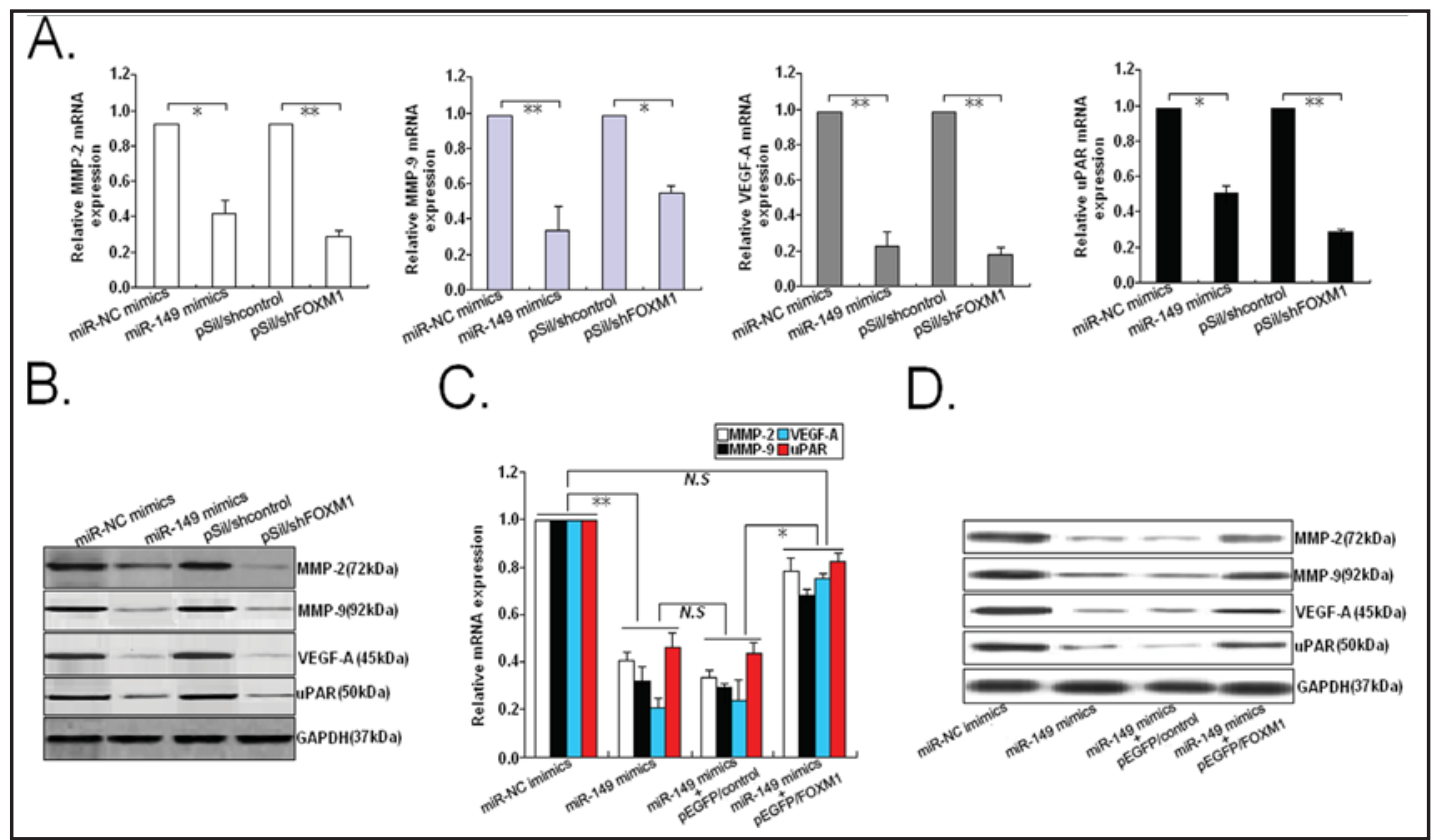

Fig. 8. miR-149 significantly downregulates the expression of MMP-2, MMP-9, VEGF-A and uPAR in CRC cells by targeting FOXM1. (A) qRT-PCR detection of the mRNA expression levels of MMP-2, MMP-9, VEGF-A and UPAR in HCT116 cells transiently transfected with miR-149 (or miR-NC) mimics or stably transfected with pSil/shFOXM1 (or pSil/shcontrol) vector. (B) Western blotting detection of the mRNA expression levels of MMP-2, MMP-9, VEGF-A and UPAR in HCT116 cells transiently transfected with miR-149 (or miR-NC) mimics or stably transfected with pSil/shFOXM1 (or pSil/shcontrol) vector. (C) qRT-PCR detection of the mRNA expression levels of MMP-2, MMP-9, VEGF-A and UPAR in miR-NC or miR-149 mimics-transfected HCT116 cells or HCT116 cells co-transfected with miR-149 mimics and pEGFP/control or pEGFP/FOXM1 vector. (D) Western blotting detection of the mRNA expression levels of MMP-2, MMP-9, VEGF-A and uPAR in miR-NC or miR-149 mimics-transfected HCT116 cells or HCT116 cells co-transfected with miR-149 mimics and pEGFP/control or pEGFP/FOXM1 vector. GAPDH was used as an internal control. Results represent the average of three independent experiments (mean \pm SD). ${ }^{*} P<0.05$, ${ }^{*} P<0.01$. N.S: not significance versus control.

of FOXM1 could reverse the growth inhibition in HCT116 cells induced by miR-149 mimics (Fig. 7B). Importantly, overexpression of FOXM1 could partially reverse the decreased capacity of migration and invasion in HCT116 cells induced by miR-149 mimics (Fig. 7C and D). Thus, these findings further show that FOXM1 is a functional target of miR-149 in CRC cells.

Upregulation of miR-149 inhibits expression of MMPs, VEGF-A and UPAR in CRC cells by downregulating FOXM1

Then, we explored the underlying molecular mechanisms involved in miR-149/FOXM1promoting migration and invasion of CRC cells. Previously, FOXM1 has been reported to promote the expression of many factors that are involved in the degradation of extra cellular matrix and angiogenesis such as UPAR, MMP-2, MMP-9, and vascular endothelial growth factor A (VEGF-A) [16, 17]. Thus, qRT-PCR and Western blotting assays were performed to detect the effects of miR-149 or FOXM1 expression on the mRNA and protein expression of MMP-2, MMP-9, VEGF-A and UPAR in CRC cells. It was observed that the mRNA expression levels of MMP-2, MMP-9, VEGF-A and uPAR in miR-149 mimics or pSil/shFOXM1-transfected HCT116 cells were significantly downregulated in comparison with miR-NC mimics or pSil/shcontrol-transfected cells (Fig. 8A). Likewise, the expression levels of those proteins in miR-149 mimics or pSil/shFOXM1-transfected HCT116 cells were also reduced in comparison with miR-NC mimics or pSil/shcontrol-transfected cells (Fig. 8B). Furthermore, 


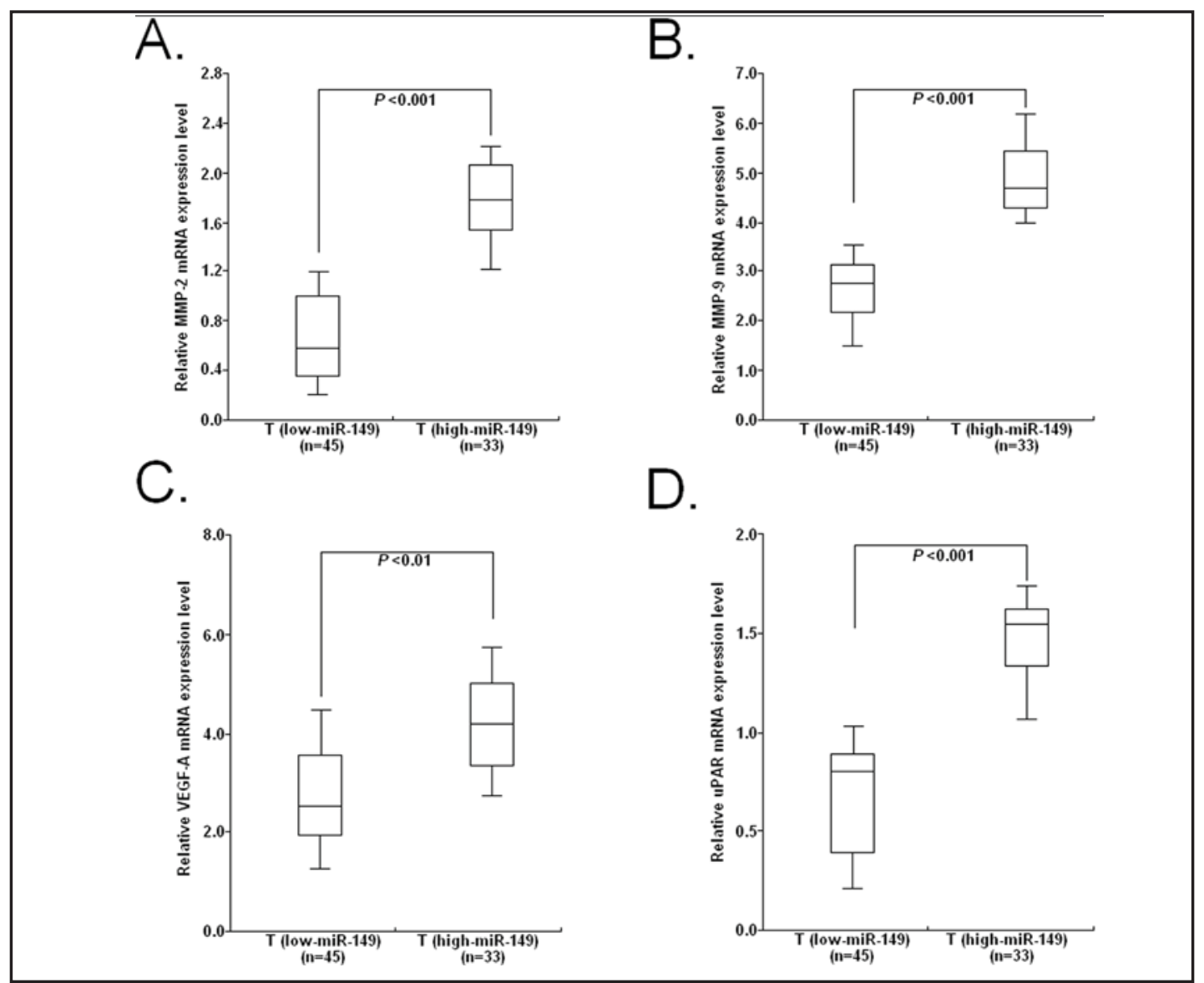

Fig. 9. qRT-PCR detection of the mRNA expression of MMP-2, MMP-2, VEGF-A and uPAR in CRC tissues. (A) qRT-PCR detection of MMP-2 mRNA expression in CRC tissues with low miR-149 expression $(n=45)$ and CRC tissues with high miR-149 expression ( $\mathrm{n}=33$ ). (B) qRT-PCR detection of MMP-9 mRNA expression in CRC tissues with low miR-149 expression ( $\mathrm{n}=45$ ) and CRC tissues with high miR-149 expression ( $\mathrm{n}=33$ ). (C) qRTPCR detection of VEGF-A mRNA expression in CRC tissues with low miR-149 expression ( $\mathrm{n}=45)$ and CRC tissues with high miR-149 expression (n=33). (D) qRT-PCR detection of uPAR mRNA expression in CRC tissues with low miR-149 expression $(n=45)$ and CRC tissues with high miR-149 expression $(n=33)$. GAPDH was used as an internal control. Results represent the average of three independent experiments (mean \pm SD).

overexpression of FOXM1 could reverse the changes of MMP-2, MMP-9, VEGF-A and UPAR mRNA and protein in HCT116 cells induced by miR-149 upregulation (Fig. 8C and D). Furthermore, we performed to detect the mRNA expression of above genes in CRC tissues, and showed that relative mRNA expression levels of MMP-2, MMP-9, VEGF-A and uPAR in CRC tissues with low miR-149 expression $(n=45)$ were significantly higher than those in tissues with high miR-149 expression (n=33) (Fig. 9A-D). These data showed that miR-149 regulates the expression of MMPs, VEGF-A and UPAR in CRC through downregulating FOXM1 expression.

FOXM1, upregulated in metastatic CRC tissues, is inversely associated with miR-149 expression

To investigate the importance of FOXM1 in primary CRC, we compared the level of FOXM1 expression $78 \mathrm{CRC}$ tissue samples and 20 normal colon tissues, which is normalized to GAPDH. The relative expression level of FOXM1 mRNA was significantly higher in CRC tissues compared to the normal colon tissues $(P<0.01$; Fig. 10A). Also, in comparison with 20 paired normal colon tissues, the expression of the FOXM1 mRNA was significantly higher 


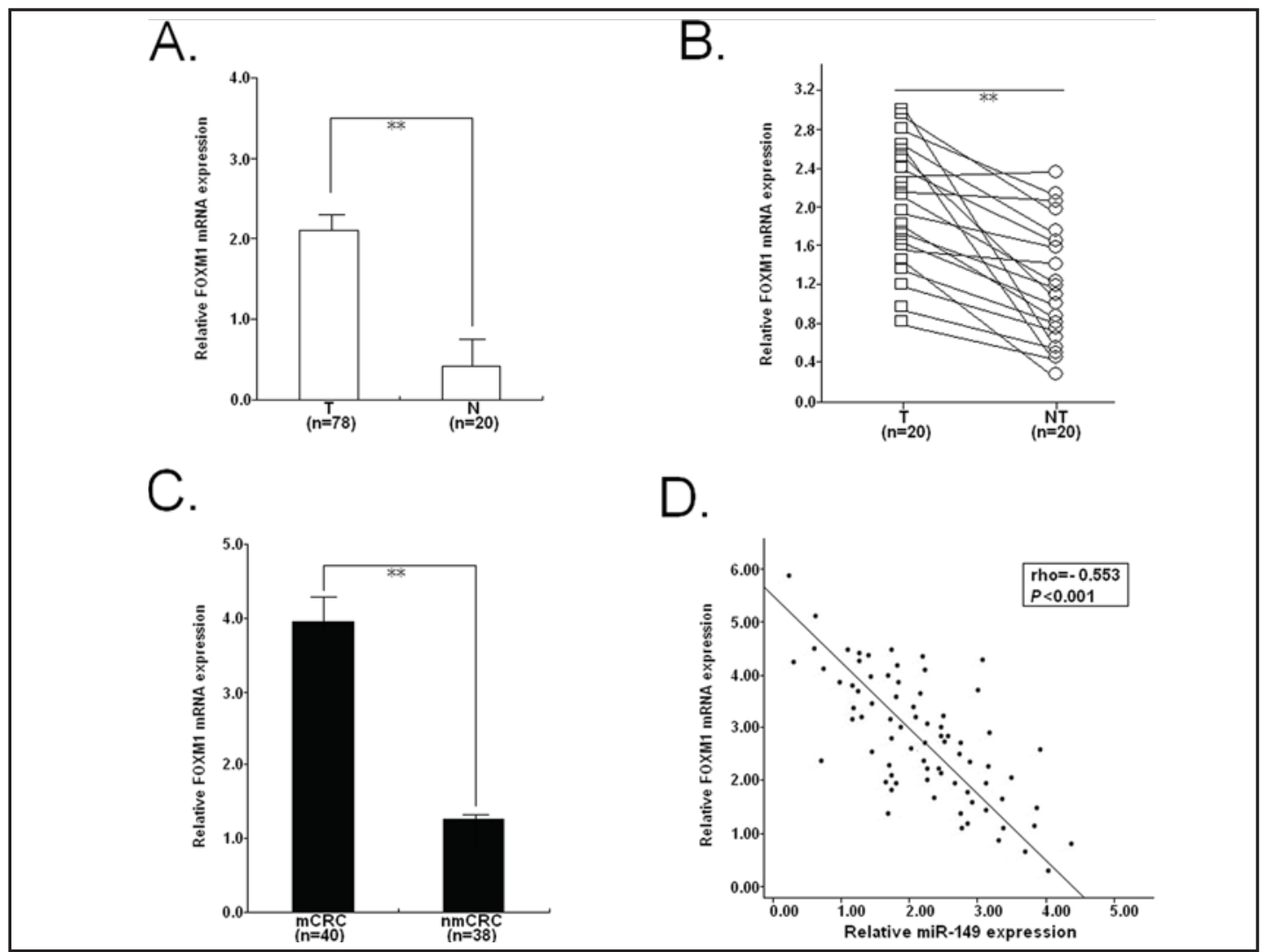

Fig. 10. FOXM1 is significantly upregulated in CRC tissues and inversely correlated with miR-149 expression. (A) qRT-PCR detection of relative FOXM1 mRNA expression in CRC tissues ( $\mathrm{n}=78$ ) and normal colon tissues (n=20). (B) qRT-PCR detection of relative FOXM1 mRNA expression in 20 paired of CRC and the adjacent normal colon tissues. (C) qRT-PCR detection of relative FOXM1 mRNA expression in CRC tissues with lymph node or distant metastasis ( $\mathrm{mCRC}, \mathrm{n}=40$ ) or without metastasis ( $\mathrm{nmCRC}, \mathrm{n}=38$ ). (D) A statistically significant inverse correlation between miR-149 and FOXM1 mRNA expression levels in 78 CRC tissues (Spearman's correlation analysis, $r=-0.553 ; P<0.001$ ). GAPDH was used as an internal control. Results represent the average of three independent experiments (mean $\pm \mathrm{SD}$ ). Corresponding $P$ values analyzed by Spearman correlation test are indicated. ${ }^{*} P<0.05$, ${ }^{* *} P<0.01$.

in CRC tissues $(P<0.01$; Fig. 10B). Meanwhile, we detected the expression of FOXM1 in CRC tissues with or without metastasis, and showed that the expression of the FOXM1 mRNA in CRC tissues with lymph node or distant metastasis (mCRC, $n=47$ ) was markedly higher than that in CRC tissues without metastasis (nmCRC, $n=31)(P<0.01$; Fig. 10C). Then, we evaluated the correlation between FOXM1 mRNA and miR-149 expression in 78 primary CRC tissues, and showed that expression of FOXM1 mRNA and miR-149 exhibited a significant inverse correlation as calculated by Pearson correlation (rho $=-0.553, P<0.001$; Fig. 10D). This result further confirmed that miR-149 targets to FOXM1 in CRC.

\section{Discussion}

Metastasis has become the major cause of death in CRC patients, and this metastatic process is highly inefficient and comprises multiple sequential steps [18]. Many genetic factors relevant in this process have already been identified, but the epigenetic factors underlying each step still remain obscure. Recently, miRNAs are recognised as master regulators of gene networks and play important roles in tumourigenesis, it is no surprise 


\section{Cellular Physiology and Biochemistry}

Cell Physiol Biochem 2015;35:499-515

\begin{tabular}{l|l}
\hline DOI: $10.1159 / 000369715$ & (C) 2015 S. Karger AG, Basel
\end{tabular}

www.karger.com/cpb

Xu et al.: MiR-149 Inhibits Tumor Cell Migration and Invasion by Targeting FOXM1 in CRC

that microRNAs have recently been demonstrated to have central roles during metastasis $[19,20]$. The correlations of dysregulated miRNAs with CRC metastasis are increasingly reported. By analysis of the miRNA expression profiles of colorectal cancer tissues with liver metastasis and their non-metastatic counterparts using microRNA microarrays and further confirmed by quantitative RT-PCR, Lin' et alshow that 28 miRNAs are differentially expressed in the colorectal carcinomas with liver metastasis compared to the non-metastatic counterparts [21]. Wang and his colleagues reported that downregulation of miR-195 correlates with lymph node metastasis and poor prognosis in colorectal cancer [22]. Wang' et al also found that serum microRNA-29a is a promising novel marker for early detection of colorectal liver metastasis [23]. The metastasis-inhibiting miRNAs (miR-342, 320a, 135a, miR-139, etc) in CRC are identified [24-27], while some metastasis-promoting miRNAs (miR-499-5p, miR-103/107, miR-155, etc) are also reported [28-30]. Has-miR-149, located on chromosome 2 , has proven to be an essential miRNA implicated in the development and the progression of human malignancies. MiR-149 has been reported to function as a tumor suppressor in human cancers. For instance, Wang' et al showed that miR-149 inhibits proliferation and cell cycle progression through the targeting of ZBTB2 in human gastric cancer [9]. Pan and his colleagues reported that miR-149 inhibits proliferation and invasion of glioma cells via blockade of AKT1 signaling [8]. MiR-149 is reported to suppress integrin signaling and breast cancer metastasis [31], but miR-149 promotes epithelial-mesenchymal transition and invasion in nasopharyngeal carcinoma cells [32]. The roles of miR-149 in CRC metastasis are still unclear and need to be further elucidated. In this study, we showed that miR-149 is significantly downregulated in CRC tissues compared to normal colon tissues. More importantly, the expression level of miR-149 in CRC tissues with lymph node or distant metastasis is lower than that in CRC tissues without metastasis. Then, we further analyzed the clinicopathological and prognostic significance of miR-149 in CRC, and showed that low miR-149 expression was closely correlated with higher incidence of lymph node or distant metastasis, advanced TNM stage and poorer prognosis of CRC patients. Then, we further performed gain-of-function assays in HCT116 cell line (low-miR-149) and loss-of - function assays in LoVo cell line (high-miR-149). Upregulation of miR-149 inhibits growth, migration and invasion of HCT116 cells, while downregulation of miR-149 promotes growth, migration and invasion of LoVo cells. As miRNAs function by target mRNAs, identification of the target mRNAs of miR-149 will contribute to characterizing the potential molecular mechanisms of miR-149-mediated phenotypical changes in CRC cells. By three target scan databases, the 3'UTR of FOXM1 mRNA possesses a miR-149-targeted seed sequence, suggesting that FOXM1 may be a putative target of miR-149.

FOXM1 (also known as HFH-11, MPP-2, WIN, and Trident), is a typical transcription factor that belongs to the Forkhead Box family, which is evolutionarily conserved and is defined by having a common DNA-binding domain called Forkhead or winged-helix domain [33]. This protein is a important regulator of both G1-S and G2-M phases of the cell cycle and mitotic spindle integrity, and its overexpression could lead to malignant transformation and tumor development [34]. The overexpression of FOXM1 and other forkhead transcription factors is found in a variety of human cancers, and has been reported to play important roles in tumor angiogenesis, invasion, and metastasis [35]. In previous study, we have reported that FOXM1 overexpression is a molecular marker predicting increased invasive/metastatic potential of CRC and a poorer prognosis. However, the molecular mechanisms involved in FOXM1 overexpression are unknown. Here, luciferase activity assay showed that miR-149 could bind to the 3'-UTR sequence of FOXM1 mRNA. Additionally, upregulation of miR-149 led to the decreased expression of FOXM1 mRNA and protein, but silencing of miR-149 induced the opposite effects on FOXM1 expression in CRC cells. Functional analyses indicated that silencing of FOXM1 could mimic the effects of miR-149 overexpression on malignant phenotypes of CRC cells. More importantly, overexpression of FOXM1 could partially reverse the effects of miR-149 upregulation in CRC cells. Downregulation of Forkhead Box M1 transcription factor has been reported to lead to the inhibition of invasion and angiogenesis of pancreatic cancer cells by reducing the expression of MMP-2, MMP-9 and VEGF [36]. 


\section{Cellular Physiology and Biochemistry}

Cell Physiol Biochem 2015;35:499-515

\begin{tabular}{l|l}
\hline DOI: $10.1159 / 000369715$ & (C) 2015 S. Karger AG, Basel
\end{tabular}

www.karger.com/cpb

Xu et al.: MiR-149 Inhibits Tumor Cell Migration and Invasion by Targeting FOXM1 in CRC

Also, FoxM1 downregulation leads to inhibition of proliferation, migration and invasion of breast cancer cells through the modulation of extra-cellular matrix degrading factors [37]. Recently, Huang and his colleagues showed that FOXM1c contributes to pancreatic cancer development and progression by enhancing uPAR gene transcription, and thus, tumor EMT and metastasis [38]. By qRT-PCR and Western blotting assays, we also found that overexpression of miR-149 or silencing of FOXM1 could induce the decreased expression of MMP-2, MMP-9, VEGF-A and uPAR at both transcriptional and translational levels. Likewise, overexpression of FOXM1 could reverse the effects of miR-149 upregulation on those genes in CRC cells. At the same time, the expression level of FOXM1 mRNA was significantly lower in metastatatic CRC tissues compared to the non-metastatic CRC tissues, and the expression of FOXM1 was inversely correlated with miR-149 expression in CRC tissues.

In summary, downregulation of miR-149 plays critical roles in CRC metastasis, and status of miR-149 expression may be an independent prognostic factor for patients. Also, miR-149 inhibits migration and invasion of CRC cells via downregulation of MMPs, VEGF-A and UPAR, at least partially by targeting FOXM1. implying the re-expression of miR-149 will be a potential therapeutic strategy for the treatment of metastatic CRC. Since the size of tissue sample in this study is small, further investigation of a larger patient population will be necessary to confirm the associations of miR-149 and its target FOXM1 with CRC metastasis. Moreover, as one miRNA could regulate multiple target genes and one gene could also be regulated by multiple miRNAs, whether miR-149 might regulate other target genes which are involved in CRC metastasis needs to be further elucidated.

\section{Acknowledgements}

This work was supported by grants from the National Natural Science Foundation of China (No.81272394). Thanks to every one of the Department of Surgery or Pathology in Jingling Hospital for their sincere help and technique support.

\section{References}

1 Siegel R, Ma J, Zou Z, Jemal A: Cancer statistics, 2014. CA Cancer J Clin 2014;64:9-29.

- Weitz J, Koch M, Debus J, Hohler T, Galle PR, Buchler MW: Colorectal cancer. Lancet 2005;365:153-165.

3 Bartel DP: MicroRNAs: genomics, biogenesis, mechanism, and function. Cell 2004;116:281-297.

4 Moreno-Moya JM, Vilella F, Simón C: MicroRNA: key gene expression regulators. Fertil Steril 2014;101:1516-1523.

5 Adams BD, Kasinski AL, Slack FJ: Aberrant Regulation and Function of MicroRNAs in Cancer.Curr Biol 2014;24:R762-R776.

6 Bouyssou JM, Manier S, Huynh D, Issa S, Roccaro AM, Ghobrial IM: Regulation of microRNAs in cancer metastasis. Biochim Biophys Acta 2014;1845:255-265.

-7 Ke Y, Zhao W, Xiong J, Cao R: miR-149 Inhibits Non-Small-Cell Lung Cancer Cells EMT by Targeting FOXM1. Biochem Res Int 2013;2013:506731.

$>8$ Pan SJ, Zhan SK, Pei BG, Sun QF, Bian LG, Sun BM: MicroRNA-149 inhibits proliferation and invasion of glioma cells via blockade of AKT1 signaling. Int J Immunopathol Pharmacol 2012;25:871-881.

-9 Wang Y, Zheng X, Zhang Z, Zhou J, Zhao G, Yang J, Xia L, Wang R, Cai X, Hu H, Zhu C, Nie Y, Wu K, Zhang D, Fan D: MicroRNA-149 inhibits proliferation and cell cycle progression through the targeting of ZBTB2 in human gastric cancer. PLoS One 2012;7:e41693.

10 Wang F, Ma YL, Zhang P, Shen TY, Shi CZ, Yang YZ, Moyer MP, Zhang HZ, Chen HQ, Liang Y, Qin HL: SP1 mediates the link between methylation of the tumour suppressor miR-149 and outcome in colorectal cancer. J Pathol 2013;229:12-24.

11 Vinci S, Gelmini S, Mancini I, Malentacchi F, Pazzagli M, Beltrami C, Pinzani P, Orlando C: Genetic and epigenetic factors in regulation of microRNA in colorectal cancers. Methods 2013;59:138-146. 


\section{Cellular Physiology and Biochemistry}

Cell Physiol Biochem 2015;35:499-515

\begin{tabular}{l|l}
\hline DOI: $10.1159 / 000369715$ & (C) 2015 S. Karger AG, Basel
\end{tabular}

www.karger.com/cpb

Xu et al.: MiR-149 Inhibits Tumor Cell Migration and Invasion by Targeting FOXM1 in CRC

-12 Du W, Ma XL, Zhao C, Liu T, Du YL, Kong WQ Wei BL, Yu JY, Li YY, Huang JW, Li ZK, Liu L: Associations of single nucleotide polymorphisms in miR-146a, miR-196a, miR-149 and miR-499 with colorectal cancer susceptibility. Asian Pac J Cancer Prev 2014;15:1047-1055.

13 Ke Y, Zhao W, Xiong J, Cao R: miR-149 Inhibits Non-Small-Cell Lung Cancer Cells EMT by Targeting FOXM1. Biochem Res Int 2013;2013:506731.

14 Koo CY, Muir KW, Lam EW: FOXM1: From cancer initiation to progression and treatment. Biochim Biophys Acta 2012;1819:28-37.

15 Chu XY, Zhu ZM, Chen LB, Wang JH, Su QS, Yang JR, Lin Y, Xue LJ, Liu XB, Mo XB: FOXM1 expression correlates with tumor invasion and a poor prognosis of colorectal cancer. Acta Histochem 2012;114:755762 .

16 Huang C, Xie D, Cui J, Li Q, Gao Y, Xie K: FOXM1c promotes pancreatic cancer epithelial-to-mesenchymal transition and metastasis via upregulation of expression of the urokinase plasminogen activator system. Clin Cancer Res 2014;20:1477-1488.

-17 Wen N, Wang Y, Wen L, Zhao SH, Ai ZH, Wang Y, Wu B, Lu HX, Yang H, Liu WC, L: Overexpression of FOXM1 predicts poor prognosis and promotes cancer cell proliferation, migration and invasion in epithelial ovarian cancer. J Transl Med 2014;12:134.

-18 Wanebo HJ, LeGolvan M, Paty PB, Saha S, Zuber M, D‘Angelica MI, Kemeny NE: Meeting the biologic challenge of colorectal metastases. Clin Exp Metastasis 2012;29:821-839.

19 Bouyssou JM, Manier S, Huynh D, Issa S, Roccaro AM, Ghobrial IM: Regulation of microRNAs in cancer metastasis. Biochim Biophys Acta 2014;1845:255-265.

20 Cheng Q Yi B, Wang A, Jiang X: Exploring and exploiting the fundamental role of microRNAs in tumor pathogenesis. Onco Targets Ther 2013;6:1675-1684.

21 Lin M, Chen W, Huang J, Gao H, Ye Y, Song Z, Shen X: MicroRNA expression profiles in human colorectal cancers with liver metastases. Oncol Rep 2011;25:739-747.

22 Wang X, Wang J, Ma H, Zhang J, Zhou X: Downregulation of miR-195 correlates with lymph node metastasis and poor prognosis in colorectal cancer. Med Oncol 2012;29:919-927.

-23 Wang LG, Gu J: Serum microRNA-29a is a promising novel marker for early detection of colorectal liver metastasis. Cancer Epidemiol 2012;36:e61-e67.

24 Wang H, Wu J, Meng X, Ying X, Zuo Y, Liu R, Pan Z, Kang T, Huang W: MicroRNA-342 inhibits colorectal cancer cell proliferation and invasion by directly targeting DNA methyltransferase 1 . Carcinogenesis 2011;32:1033-1042.

25 Zhang Y, He X, Liu Y, Ye Y, Zhang H, He P, Zhang Q, Dong L, Liu Y, Dong J: microRNA-320a inhibits tumor invasion by targeting neuropilin 1 and is associated with liver metastasis in colorectal cancer. Oncol Rep 2012;27:685-694.

-26 Zhou W, Li X, Liu F, Xiao Z, He M, Shen S, Liu S: MiR-135a promotes growth and invasion of colorectal cancer via metastasis suppressor 1 in vitro. Acta Biochim Biophys Sin (Shanghai) 2012;44:838-846.

27 Shen K, Liang Q, Xu K, Cui D, Jiang L, Yin P, Lu Y, Li Q, Liu J: MiR-139 inhibits invasion and metastasis of colorectal cancer by targeting the type I insulin-like growth factor receptor. Biochem Pharmacol 2012;84:320-330.

-28 Liu X, Zhang Z, Sun L, Chai N, Tang S, Jin J, Hu H, Nie Y, Wang X, Wu K, Jin H, Fan D: MicroRNA-499-5p promotes cellular invasion and tumor metastasis in colorectal cancer by targeting FOXO4 and PDCD4. Carcinogenesis 2011;32:1798-1805.

29 Chen HY, Lin YM, Chung HC, Lang YD, Lin CJ, Huang J, Wang WC, Lin FM, Chen Z, Huang HD, Shyy JY, Liang JT, Chen RH: miR-103/107 promote metastasis of colorectal cancer by targeting the metastasis suppressors DAPK and KLF4. Cancer Res 2012;72:3631-3641.

-30 Zhang GJ, Xiao HX, Tian HP, Liu ZL, Xia SS, Zhou T: Upregulation of microRNA-155 promotes the migration and invasion of colorectal cancer cells through the regulation of claudin-1 expression. Int J Mol Med 2013;31:1375-1380.

-31 Chan SH, Huang WC, Chang JW, Chang KJ, Kuo WH, Wang MY, Lin KY, Uen YH, Hou MF, Lin CM, Jang TH, Tu CW, Lee YR, Lee YH, Tien MT, Wang LH: MicroRNA-149 targets GIT1 to suppress integrin signaling and breast cancer metastasis. Oncogene 2014;33:4496-4507.

-32 Luo Z, Zhang L, Jiang C, Dai Y, Liu X, Zheng Y, Yu H, Xiang J, Li G: miR-149 promotes epithelial-mesenchymal transition and invasion in nasopharyngeal carcinoma cells. Zhong Nan Da Xue Xue Bao Yi Xue Ban 2011;36:604-609. 


\section{Cellular Physiology Cell Physiol Biochem 2015;35:499-515 and Biochemistry

Katoh M, Katoh M: Human FOX gene family (Review). Int J Oncol 2004;25:1495-1500.

-34 Halasi M, Gartel AL: Targeting FOXM1 in cancer. Biochem Pharmacol 2013;85:644-652.

-35 Gomes AR, Zhao F, Lam EW: Role and regulation of the forkhead transcription factors FOXO3a and FOXM1 in carcinogenesis and drug resistance. Chin J Cancer 2013;32:365-370.

-36 Wang Z, Banerjee S, Kong D, Li Y, Sarkar FH: Down-regulation of Forkhead Box M1 transcription factor leads to the inhibition of invasion and angiogenesis of pancreatic cancer cells. Cancer Res 2007;67:82938300.

-37 Ahmad A, Wang Z, Kong D, Ali S, Li Y, Banerjee S, Ali R, Sarkar FH: FoxM1 down-regulation leads to inhibition of proliferation, migration and invasion of breast cancer cells through the modulation of extracellular matrix degrading factors. Breast Cancer Res Treat 2010;122:337-346.

38 Huang C, Xie D, Cui J, Li Q, Gao Y, Xie K: FOXM1c promotes pancreatic cancer epithelial-to-mesenchymal transition and metastasis via upregulation of expression of the urokinase plasminogen activator system. Clin Cancer Res 2014;20:1477-1488. 